\title{
Graph transformations preserving the stability number
}

\author{
Benjamin Lévêque, Dominique de Werra \\ Ecole Polytechnique Fédérale de Lausanne \\ Lausanne, Switzerland
}

May 27, 2008

\begin{abstract}
In this note we analyse the relations between several graph transformations that were introduced to be used in procedures determining the stability number of a graph. We show that all these transformations can be decomposed into a sequence of edge deletions and twin deletions. We also show how some of these transformations are related to the notion of even pair introduced to color some classes of perfect graphs. Then, some properties of edge deletion and twin deletion are given and a conjecture is formulated about the class of graphs for which these transformations can be used to determine the stability number.
\end{abstract}

\section{Introduction}

A set of pairwise non-adjacent vertices in a graph $G$ is called a stable set. The maximum size of a stable set in a graph $G$ is called the stability number and is noted $\alpha(G)$. Given a vertex $v$ of a graph, let $N(v)$ denote the set of vertices that are adjacent to $v$. The closed neighborhood $N[v]$ of a vertex $v$ is $N(v) \cup\{v\}$. In a specific graph $H$, we note $N_{H}(v)$ the neighborhood of $v$ and $N_{H}[v]$ its closed neighborhood. A clique in a graph is a complete subgraph. The triangle is the clique of size three. The maximum size of a clique in a graph $G$ is called the clique number and is noted $\omega(G)$. The complement $\bar{G}$ of a graph $G$ is the graph obtained by removing all the edges that were in $G$ and adding all the edges that were not in $G$. For a vertex $v$, the set $N_{\bar{G}}(v)$ will be simply noted $\bar{N}(v)$. Clearly, $\alpha(G)=\omega(\bar{G})$ for every graph $G$. The chromatic number of a graph $G$ is the minimum number of colors that can be assigned to the vertices of a graph in such a way that two adjacent vertices receive two distinct colors. This number is noted $\chi(G)$. Clearly, $\omega(G) \leq \chi(G)$, for every graph $G$. The decision problems associated to determining $\alpha, \omega$ and $\chi$ are known to be NP-complete [13].

A chordless path is a set of vertices $v_{1}, \ldots, v_{k}$, such that for $1 \leq i \leq k-1$, vertex $v_{i}$ is adjacent to $v_{i+1}$ and there is no other edge between these vertices. Such a path will sometimes be denoted $v_{1} \cdots-v_{k}$. The length of a path is the number of its edges. A 
path is odd if it has odd length and even otherwise. Let $P_{k}$ denote the chordless path on $k$ vertices (of length $k-1$ ). A hole is a chordless cycle with at least five vertices. An antihole is the complement of a hole. A hole or an antihole is odd if it has an odd number of vertices and even otherwise. The square is the chordless cycle of length four.

We will introduce several graph transformations preserving $\alpha$ in Section 2 and $\omega$ is Section 3. We show how these transformations are linked to each other and how they can be decomposed in two basic transformations that are the edge deletion and twin deletion. In Section 4, we discuss how these simple transformations may be combined to compute $\alpha$.

\section{Transformations preserving the stability number}

Many graph transformations have been defined in order to determine the size of a maximum stable set in special classes of graphs. Here we shall concentrate on transformations which simplify the graph $G$ by removing vertices and/or edges, while keeping the same value of $\alpha(G)$. Other operations have been introduced for computing $\alpha(G)$ by transforming the graph $G$ into another graph $G^{\prime}$ with $\alpha\left(G^{\prime}\right)=\alpha(G)-p$ (where $p$ is a fixed integer). The struction, derived initially with pseudo boolean arguments (see [10]), is such an example. We shall not discuss those kind of transformations here, but refer the reader to [1].

\subsection{Simplicial, neighborhood and magnet reductions}

A vertex $v$ is called simplicial if $N(v)$ is a clique. If $v$ is a simplicial vertex, then the deletion of $N(v)$ does not change the stability number. This transformation is called the simplicial reduction. A graph is chordal if it contains no hole and no square as an induced subgraph. By a theorem of Dirac [9], every chordal graph contains a simplicial vertex. So, the simplicial vertex reduction can be used to determine the stability number of a chordal graph. This can be done in linear time by using algorithms Lexicographic Breath First Search [24] or Maximum Cardinality Search [25].

If $a$ and $b$ are two adjacent vertices such that $N(b) \subseteq N[a]$, then the deletion of $a$ does not change the stability number. This transformation is called the neighborhood reduction. The neighborhood reduction generalizes the simplicial vertex reduction. If $v$ is a simplicial vertex, then the deletion of $N(v)$ can be viewed as a sequence of neighborhood reductions for each vertex $a \in N(v)$, as $N(v) \subseteq N[a]$. In [14], the neighborhood reduction has been used to transform any circular arc graph into a canonical form for which the stability number could be easily determined.

It was shown in [10] that finding the stability number of a graph could be reduced to the problem of maximizing a pseudo-boolean expression called a posiform (i.e, a polynomial of 0,1 variables $x_{i}$ and their complements $\overline{x_{i}}=1-x_{i}$, where all coefficients of monomials are positive). Conversely, to any maximization problem of a pseudoboolean function, we can associate an equivalent problem of finding a maximum stable 
set in a graph. In some cases, algebraic manipulations in a posiform can be devised to simplify the posiform without affecting its maximum value. In this way, a purely graph theoretical transformation called the magnet reduction was discovered.

A magnet [16] consists of two adjacent vertices $a, b$ such that $N(a) \backslash N(b)$ is completely linked to $N(b) \backslash N(a)$. If $a$ and $b$ form a magnet, then the deletion of $a$ and of all the edges between $b$ and $N(b) \backslash N(a)$ does not change the stability number. This transformation is called the magnet reduction. Clearly, the magnet reduction generalizes the neighborhood reduction. If $a$ and $b$ are two adjacent vertices such that $N(b) \subseteq N[a])$, then $N(b) \backslash N[a]=$ $\emptyset$, so $N(a) \backslash N(b)$ is completely linked to $N(b) \backslash N(a)$. Deleting $a$ corresponds to the magnet reduction as $N(b) \backslash N[a]=\emptyset$.

\section{$2.2 \quad$ Edge and twin deletions}

If $a, b, c$ are three vertices such that $a-b-c$ is a chordless path and $N(a) \subseteq N(b) \cup N(c)$, then the deletion of the edge $b c$ does not change the stability number. This transformation is called the edge deletion [4].

As remarked in [1], the magnet reduction can be viewed as a sequence of edge deletions followed by one neighborhood reduction. If $a, b$ are two adjacent vertices such that $N(a) \backslash N(b)$ is completely linked to $N(b) \backslash N(a)$, then for each $c$ in $N(b) \backslash N[a]$, we have that $a-b-c$ is a chordless path and $N(a) \subseteq N(b) \cup N(c)$, so edge $b c$ can be deleted (edge deletion). When $N(b) \backslash N[a]$ is empty, then $N(b) \subseteq N[a]$ and $a$ can be deleted (neighborhood reduction).

Two adjacent vertices $a, b$ are twins if $N[a]=N[b]$. If $a, b$ are twins, then the deletion of $a$ does not change the stability number. We call this transformation the twin deletion. The twin deletion has been used in [5] to determine the stability number of cographs. Clearly, twin deletion is a special case of neighborhood reduction, but the neighborhood reduction can be viewed as a sequence of edge deletions followed by one twin deletion. If $a$ and $b$ are two adjacent vertices such that $N(a) \subseteq N[b]$, then for each $c \in N(b) \backslash N[a]$ we have that $a-b-c$ is a chordless path and $N(a) \subseteq N(b) \cup N(c)$, so edge $b c$ can be deleted (edge deletion). When $N(b) \backslash N[a]$ is empty, then $N[a]=N[b]$ and $a$ can be deleted (twin deletion).

Now we can decompose the magnet reduction in the two simple transformations that are edge and twin deletions:

Proposition 1 The magnet reduction is a sequence of edge deletions followed by one twin deletion.

Proof. By the previous remarks, the magnet reduction is a sequence of edge deletions followed by one neighborhood reduction, and the neighborhood reduction is a sequence of edge deletions followed by one twin deletion. So, the magnet reduction is a sequence of edge deletions followed by one twin deletion. 


\section{Transformations preserving the clique number}

Some other graph transformations have been defined to determine the chromatic number of some subclasses of perfect graphs. The class of perfect graphs [2] has been defined as the class of graphs $G$ such that for every induced subgraph $H$ of $G$, we have $\chi(H)=$ $\omega(H)$. The strong perfect graph theorem [6] asserts that a graph is perfect if and only if it contains no odd hole and no odd antihole. The class of perfect graphs appears to be a general class of graphs in which the problem of determining the chromatic number can be solved in polynomial time [15] with the the ellipsoid method [17]. But the problem of finding a purely combinatorial algorithm for determining $\chi$ is still open. We will give next graph transformations that appear to be useful to solve this problem.

\subsection{Even pair contraction}

If $a, b$ are two non adjacent vertices in a graph $G$, the contraction of $a$ and $b$ consists in deleting $a$ and $b$ and adding a new vertex called $a b$ that is adjacent to every vertex of $N(a) \cup N(b)$. An even pair [22] is a pair of non adjacent vertices such that there is no odd chordless path between them. It has been shown that the even pair contraction preserves the chromatic number and the clique number in any graph (not necessarily perfect) [12]. The notion of even pair plays a major role in the class of perfect graphs. It has been used to find a substantial shortcut [7] in the proof of the strong perfect graph theorem [6]. Moreover it can be used to determine $\chi$ with combinatorial tools in many subclasses of perfect graphs [11].

A graph is called contractile [3] if it can be reduced to a clique by a sequence of even pair contraction. As the even pair contraction preserves $\chi$ and $\omega$, one can determine the chromatic number and clique number of a contractile graph as soon as one has a sequence of even pair contractions that transforms the graph into a clique. A graph is called perfectly contractile [3] if all its induced subgraphs are contractile.

A prism is a graph that consists of two vertex-disjoint triangles and three vertexdisjoint paths between them, with no other edge than those in the two triangles and in the three paths. When odd holes are forbidden the length of the three paths of a prism must have the same parity. A prism is odd, if the length of the three paths is odd, and even if the length of the three paths is even.

The following conjecture tries to characterize the class of perfectly contractile graphs by forbidden induced subgraphs:

Conjecture 1 ([23]) A graph is perfectly contractile if and only if it contains no odd hole, no antihole and no odd prism.

A weaker form of this conjecture has been proved:

Theorem 1 ([21]) A graph that contains no odd hole, no antihole and no prism is perfectly contractile. 
An $\mathcal{O}\left(n^{2} m\right)$ algorithm [20] has been deduced from the proof of Theorem 1 to compute the chromatic and clique number of graphs with no odd hole, no antihole and no prism.

A $P_{4}$-free pair is a pair of non adjacent vertices such that there is no chordless path of length three between them. This generalization of even pair has been introduced by the first author in [19]. To prove that the even pair contraction preserves $\omega$, as done in [12], there is no need to exclude odd chordless paths of length $\geq 5$ and a corollary of this remark is that contracting a $P_{4}$-free pair preserves the clique number [19].

Unfortunately, the $P_{4}$-free pair contraction does not preserve the chromatic number as one can remark by contracting the end vertices of a $P_{6}$. But we can nevertheless define classes of contractile graphs as in [3]. A graph is called $P_{4}$-free-contractile [19] if it can be reduced to a clique by a sequence of $P_{4}$-free pair contractions. As the $P_{4}$-free pair contraction preserves $\omega$, and for a clique we have $\chi$ equals $\omega$, one can determine the chromatic and clique numbers of a $P_{4}$-free-contractile graph as soon as one has a sequence of $P_{4}$-free pair contractions that transforms the graph into a clique [19]. A graph is called perfectly $P_{4}$-free-contractile [19] if all its induced subgraphs are $P_{4}$-freecontractile. It is conjectured in [19] that a graph is perfectly $P_{4}$-free-contractile if and only if it contains no odd hole and no antihole.

At this stage, one may wonder whether there are some connections between the various transformations preserving $\alpha$ described in section 2 and the contractions mentioned above which were devised independently using entirely different arguments. It turns out that we have the following :

Proposition $2 A$ A $P_{4}$-free pair contraction in a graph $G$ is precisely a magnet reduction in the complement $\bar{G}$.

Proof. The vertices $a$ and $b$ form a $P_{4}$-free pair in a graph $G$, if and only if, in $\bar{G}$, they are adjacent and they are not the middle vertices of a $P_{4}$, so, if and only if, they are adjacent and $\bar{N}(a) \backslash \bar{N}(b)$ is completely linked to $\bar{N}(b) \backslash \bar{N}(a)$. In $\bar{G}$, the deletion of $a$ and of all the edges between $b$ and $\bar{N}(b) \backslash \bar{N}(a)$ coincide with the contraction of $a, b$ in $G$.

Figure 1 summarizes the relations between the graph transformations that we have considered. An edge $A \rightarrow B$ means that the transformation $A$ is a special case of transformation $B$. A transformation $[A]$ is the transformation $A$ considered in the complement, and $A^{*}+B$ means a sequence of $A$ 's followed by one $B$.

\subsection{Odd pair insertion}

Asserting that an even pair contraction does not change the chromatic number amounts to saying that there exists an optimum coloring where the two contracted vertices receive the same color. In a symetric way, one may ask when it is legitimate to say that there exists an optimum coloring where two non adjacent vertices $a, b$ have different colors. This would mean that we can introduce an edge $a b$ without changing the chromatic number. Two non adjacent vertices $a, b$ form an odd pair [11] if there is no even chordless 


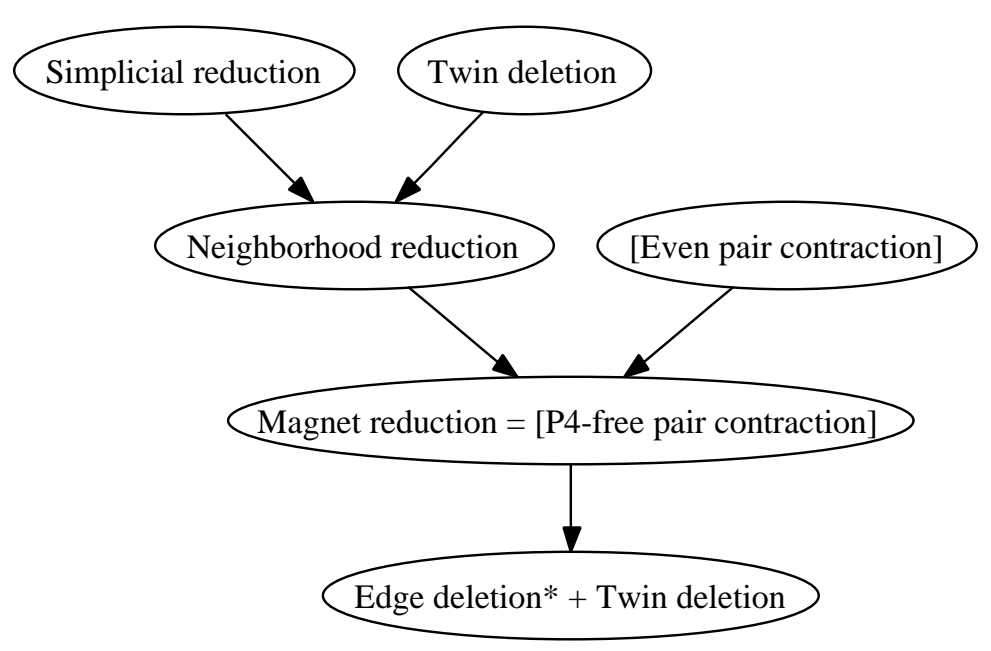

Figure 1: Relations between considered graph transformations

path between them. If $\omega \geq 2$, adding the edge $a b$ preserves the chromatic number and the clique number [11]. This transformation is called the odd pair insertion.

As for even pairs, on may remark that to prove that the odd pair insertion preserves $\omega$, there is no need to exclude even chordless paths of length $\geq 4$. A $P_{3}$-free pair is a pair of non adjacent vertices $a, b$ such that there is no chordless path of length two between them. If $\omega \geq 2$, adding the edge $a b$ preserves $\omega$ (but not $\chi$ as one may remark by adding an edge between the end vertices of a $P_{5}$ ). This transformation is called the $P_{3}$-free pair insertion.

Proposition 3 In a graph $G$, a $P_{3}$-free pair insertion with vertices $a$ and $b$ such that $N(a) \neq N(b)$ is a special case of edge deletion in $\bar{G}$.

Proof. Supose $a, b$ is a $P_{3}$-free pair in $G$ with $N(a) \neq N(b)$. Then, in $\bar{G}$, $a$ and $b$ are adjacent and all the vertices of $G$ are in $\bar{N}(a) \cup \bar{N}(b)$. As $N(a) \neq N(b)$ we have $\bar{N}[a] \neq \bar{N}[b]$. Then one of $\bar{N}(a) \backslash \bar{N}[b]$ or $\bar{N}(b) \backslash \bar{N}[a]$ is non empty. By symetry we can assume that there exists $c \in \bar{N}(b) \backslash \bar{N}[a]$. Then $a-b-c$ is a $P_{3}$ and $\bar{N}(c) \subseteq \bar{N}(b) \cup \bar{N}(a)$. So, deleting $a b$ in $\bar{G}$ is an edge deletion.

\section{$4 \quad$ Edge and twin deletions}

As shown in figure 1, many graph transformations that were introduced to compute $\alpha$, $\omega$ or $\chi$ are simply combinations of edge and twin deletions. 


\subsection{Edge-deletable graphs}

If one can transform a graph into a stable set by applying a sequence of edge and twin deletions, then one can determine the stability number of the original graph. It is exactly the number of vertices of the stable set that is obtained.

Proposition 4 If a graph $G$ can be transformed into a stable set by applying a sequence of edge and twin deletions, then $G$ can be transformed into a stable set by applying first a sequence of edge deletions, then a sequence of twin deletions.

Proof. We claim that a twin deletion followed by an edge deletion can be replaced by one or two edge deletions followed by a twin deletion. By repeatedly applying this, one can place all the twin deletions at the end of the sequence and get the result.

Suppose that $x$ and $y$ are twins in a graph $G$. Let $H$ the graph obtained after deleting $x$. Suppose there exists three vertices $a, b, c$ of $H$ such that $a-b-c$ is a chordless path and $N_{H}(a) \subseteq N_{H}(b) \cup N_{H}(c)$ ( $y$ is possibly one of $a, b, c$, but not $x$ ). Let $H^{\prime}$ be the graph obtained from $H$ by deleting edge $b c$.

In $G$, the three vertices $a, b, c$ form a chordless path $a$-b-c. Suppose $N(a) \nsubseteq N(b) \cup$ $N(c)$, then $x \in N(a) \backslash(N(b) \cup N(c))$. If $y=a$, then $b \in N[y]=N[x]$, a contradiction. If $y \neq a$, then $y$ is also in $N(a) \backslash(N(b) \cup N(c))$ and $b c$ cannot be deleted in $H$, a contradiction. So $N(a) \subseteq N(b) \cup N(c)$ and we can delete the edge $b c$ to obtain the graph $G^{\prime}$.

If $x, y$ are still twins in $G^{\prime}$, then we can delete $x$ to obtain $H^{\prime}$. Suppose now that $x, y$ are not twins in $G^{\prime}$. Then $y$ is one of $b, c$. If $y=b$, then the three vertices $a, x, c$ form a chordless path $a-x-c$ of $G^{\prime}$ with $N_{G^{\prime}}(a) \subseteq N_{G^{\prime}}(x) \cup N_{G^{\prime}}(c)$ so we can delete the edge $x c$, then $x$ and $y$ become twins and we can delete $x$ to obtain $H^{\prime}$. If $y=c$, then the three vertices $a, b, x$ form a chordless path $a-b-x$ of $G^{\prime}$ with $N_{G^{\prime}}(a) \subseteq N_{G^{\prime}}(b) \cup N_{G^{\prime}}(x)$ so we can delete the edge $b x$, then $x$ and $y$ become twins and we can delete $x$ to obtain $H^{\prime}$.

Proposition 5 A graphs can be reduced to a stable set by applying a sequence of twin deletions if and only if it consists of a set of disjoint cliques.

Proof. If a graph is a set of disjoint cliques that is not a stable set, then there exists a clique containing at least two vertices that are twins. We can remove one of them to get a set of disjoint cliques with strictly less vertices. This operation can be repeated until we get a stable set.

If a graph is not a set of disjoint cliques, then it contains a $P_{3}$. The graph will still contain a $P_{3}$ after any twin deletion and so it is not possible to get a stable set.

By Proposition 4 and 5, if one wants to determine the stability number of a graph by using edge and twin deletions, one can apply only edge deletion and stop when the 
graph is a disjoint set of cliques. Then, the stability number of the original graph is exactly the number of disjoint cliques when the process stops.

A graph is called edge-deletable if it can be reduced to a set of disjoint cliques by a sequence of edge deletion. A graph is called perfectly edge-deletable if all its induced subgraphs are edge-deletable.

\subsection{Forbidden induced subgraphs}

Proposition 6 A hole is not edge-deletable

Proof. Consider a hole $x_{1} \cdots-x_{k}-x_{1}$, with $k \geq 5$. Three vertices that form a $P_{3}$ must be three consecutive vertices, for example $x_{1}-x_{2}-x_{3}$. We have $x_{k} \in N\left(x_{1}\right) \backslash\left(N\left(x_{2}\right) \cup N\left(x_{3}\right)\right)$ and $x_{4} \in N\left(x_{3}\right) \backslash\left(N\left(x_{2}\right) \cup N\left(x_{1}\right)\right)$, so no edge deletion can be applied.

\section{Proposition 7 An odd antihole is not edge-deletable}

Proof. Suppose there exists an odd antihole $A$ that is edge-deletable. The stability number of $A$ is 2 , so one can delete some edges of $A$ to obtain a set of two disjoint cliques. But the vertices of odd antiholes cannot be partitioned into two cliques, a contradiction.

A consequence is the following:

Corollary 1 A perfectly edge-deletable graph contains no hole and no odd antihole.

As a corollary of Theorem 1, we can state:

Corollary 2 Every graph that contains no hole, no odd antihole, and no complement of a prism is perfectly edge-deletable.

Moreover we have:

Proposition 8 Complements of even prisms and complements of odd prisms different from $C_{6}$ are edge-deletable.

Proof. Even prisms are contractile, so complements of even prisms are edge-deletable.

Odd prisms that are different from $C_{6}$ can be reduced to the odd prism on 8 vertices by a sequence of even pair contractions by contracting two of the three paths to an edge, and the third path to a $P_{3}$. The complement of the odd prism on 8 vertices is perfectly edge-deletable as shown in Figure 2. So complements of odd prisms are edge-deletable.

The following conjecture tries to characterize the class of perfectly edge-deletable graphs by forbidden induced subgraphs: 

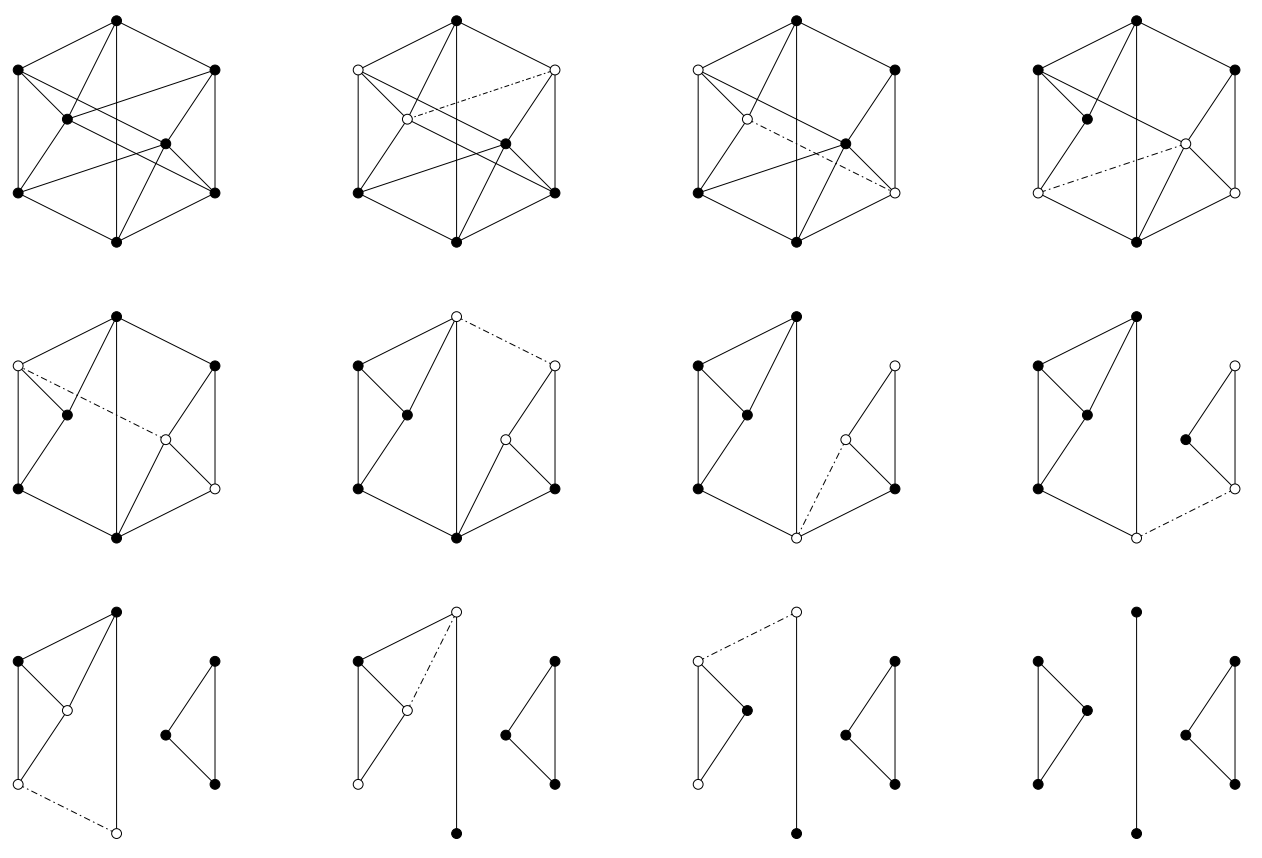

Figure 2: The complement of the odd prism on 8 vertices is edge-deletable

Conjecture 2 A graph is perfectly edge-deletable if and only if it contains no hole and no odd antihole.

Proving such a conjecture will be a substantial step in the process of finding a purely combinatorial algorithms for determining the chromatic number of perfect graphs.

A result of [8] provides the first step of a proof of conjecture 2. A chordless path $v_{1}-\cdots-v_{k}$ is simplicial if it cannot be extended to a chordless path $v_{0}-v_{1}-\cdots-v_{k}-v_{k+1}$. It is easy to see that, given a graph $G$ and an integer $k$, if every non empty induced subgraph of $G$ contains a simplicial path on at most $k$ vertices, then $G$ contains no hole of length $\geq k+3$. The converse is also true:

Theorem 2 ([8]) For all positive integers $k$, a graph contains no hole of size $\geq k+3$ if and only if all its non empty induced subgraphs contain a simplicial path on at most $k$ vertices.

Applied for $k=2$, we get the following corollary.

Corollary 3 Given a graph with no hole, either it is a set of pairwise disjoint cliques or edge deletion can be applied.

Proof. Let $G$ be a graph with no hole that is not a set of pairwise disjoint cliques. Let $H$ be a connected component of $G$ that contains a $P_{3}$. Let $H^{\prime}$ be a maximal 
(inclusionwise) induced subgraph of $H$ that contains no twins. Then $H^{\prime}$ still contains a $P_{3}$. By Theorem $2, H^{\prime}$ contains a simplicial path $P$ on at most 2 vertices.

If $|P|=1$, let $P=\{a\}$. Vertex $a$ is a simplicial vertex. Let $b \in N(a)$ (it exists as $H^{\prime}$ is connected and contains at least 3 vertices). Vertices $a, b$ are not twins and $N(a) \subseteq N[b]$, so there exists $c \in N(b) \backslash N[a]$. If $|P|=2$, let $P=\{a, b\}$. Vertices $a, b$ are not twins, so we can assume that there exists $c \in N(b) \backslash N[a]$. As $P$ is simplicial, $N(a) \backslash N[b]=\emptyset$. In both cases, vertices $a, b, c$ are such that $a-b-c$ is a chordless path and $N(a) \subseteq N(b) \cup N(c)$, so edge deletion can be applied.

This corollary shows that it is always possible to start the edge deletion method in a graph with no hole and no odd antihole. But it cannot be guaranteed that this first step will not create a hole or an odd antihole. This is for example the case for the complement of the odd prism on eight vertices: whatever edge deletion in performed, a hole is created.

\section{Comments}

We have shown that many graph transformations preserving the stability number (or equivalently the clique number in the complement) can be expressed as a sequence of edge deletions followed by one twin deletion. This is not the case for all transformations that preserve $\alpha$. For example, the BAT, defined in [18], cannot be decomposed into edge and twin deletions. We have to allow a third basic transformation called edge insertion [4] to be able to decompose the BAT. The problem of allowing edge insertion is that the reduction of a graph to a stable set by a sequence of edge deletions, edge insertions and twin deletions is not necessarly polynomial anymore. One may delete and insert the same edge many times.

It would be interesting to explore further a combination of the transformations discussed here with the other techniques like the struction to compute $\alpha$. Some preliminary experiments are reported in [1].

\section{References}

[1] G. Alexe, P.L. Hammer, V.V. Lozin, D. de Werra, Struction revisited, Discrete Appl. Math. 132 (2004) 27-46.

[2] C. Berge, Les problèmes de coloration en théorie des graphes, Publications de l'Institut de Statistiques de l'Université de Paris (1960) 123-160.

[3] M.E. Bertschi, Perfectly contractile graphs, Journal of Combinatorial Theory B 50 (1990) 222-230. 
[4] L. Butz, P.L. Hammer, D. Haussmann, Reduction methods for the vertex packing problem, Proceedings of the 17th Conference on Probability Theory, Brasov, 1982, VNU Science Press, Utrecht, 1985, 73-79.

[5] D. G. Corneil, H. Lerchs, L. Stewart Burlingham, Complement reducible graphs, Discrete Appl. Math. 3 (1981) 163-174.

[6] M. Chudnovsky, N. Robertson, P. Seymour, R. Thomas, The strong perfect graph theorem, Annals of Mathematics 164 (2006) 51-229.

[7] M. Chudnovsky, P. Seymour, Even pairs in Berge graphs, manuscript, 2007.

[8] V. Chvátal, I. Rusu, R. Sritharan, Dirac-type characterizations of graphs without long chordless cycles, Discrete Mathematics 256 (2002) 445-448.

[9] G.A. Dirac, On rigid circuit graphs, Abh. Math. Sem. Univ. Hamburg 38 (1961) $71-76$.

[10] Ch. Ebenegger, P.L. Hammer, D. de Werra, Pseudo-Boolean functions and stability of graphs, Ann. Discrete Math. 19 (1984) 83-98.

[11] H. Everett, C.M.H. de Figueiredo, C. Linhares Sales, F. Maffray, O. Porto, B.A. Reed, Even pairs, Perfect Graphs, J. L. Ramírez-Alfonsín and B. A. Reed, eds., Wiley Interscience, Chichester, UK, 2001, 67-92.

[12] J. Fonlupt, J.P. Uhry, Transformations that preserve perfectness and $h$-perfectness of graphs, Annals of Discrete Mathematics 16 (1982) 83-85.

[13] M.R. Garey, D.S. Johnson, Computers and Intractability, Freeman, San Francisco, California, 1979.

[14] M.C. Golumbic, P.L. Hammer, Stability in circular-arc graphs, J. Algorithms 9 (1988) 314-320.

[15] M. Gröstchel, L. Lovász, A. Schrijver, The ellipsoid method and its consequences in combinatorial optimization, Combinatorica 1 (1981) 169-197.

[16] P.L. Hammer, A. Hertz, On a transformation which preserves the stability number, RUTCOR Research Report 69-91, Rutgers University, 1991.

[17] L.G. Khachiyan, A polynomial algorithm in linear programming, Soviet Mathematics Doklady 20 (1979) 191-194.

[18] A. Hertz, On the use of Boolean methods for the computation of the stability number, Discrete Appl. Math. 76 (1997) 183-203.

[19] B. Lévêque, Coloring graphs : structures and algorithms, PhD Thesis, Grenoble University, 2007, http://tel.archives-ouvertes.fr/tel-00187797. 
[20] B. Lévêque, F. Maffray, B.A. Reed, N. Trotignon, Coloring Artemis graphs, manuscript, 2007.

[21] F. Maffray, N. Trotignon, A class of perfectly contractile graphs, Journal of Combinatorial Theory B 96 (2006) 1-19.

[22] H. Meyniel, A new property of critical imperfect graphs and some consequences, European Journal of Combinatorics 8 (1987) 313-316.

[23] B.A. Reed, Problem session on parity problems (Public communication), DIMACS Workshop on Perfect Graphs, Princeton University, New Jersey, 1993.

[24] D.J. Rose, R.E. Tarjan, G.S. Lueker, Algorithmic aspects of vertex elimination of graphs, SIAM Journal on Computing 5 (1976) 266-283.

[25] R.E. Tarjan, M. Yannakakis, Simple linear time algorithms to test chordality of graphs, test acyclicity of hypergraphs, and selectively reduce acyclic hypergraphs, SIAM J. Comput. 13 (1984) 566-579. 